\title{
Cycloparaphenylenes and their Catenanes - Complex Macrocycles Unveiled by Ion Mobility Mass Spectrometry
}

\author{
Wen Zhang, Ali Abdulkarim, Florian E. Golling, Hans Joachim Räder*, and Klaus Müllen*
}

\begin{abstract}
We analyze the insoluble product mixture obtained from cycloparaphenylene (CPP) synthesis via Suzuki coupling and reductive aromatization. Traditional mass spectrometry suggests a homologous series of macrocycles with 12 to 84 phenylene units. Ion mobility mass spectrometry (IMMS), however, unravels an unexpected complexity of isomers with identical chemical formula, but different topologies. Whereas macrocycles containing up to 30 phenylene units show only one structure, the homologue with 36 phenylene units forms at least four different isomers with significant molecular size differences. They can be assigned to catenanes composed of CPPs with $2 \times 18$ and $12+24$ phenylene units together with the ordinary [36]CPP macrocycle. Most likely, a trefoil knot of the CPP with 36 moieties is also present. For the first time, catenanes can be elucidated in a simple reaction mixture by analyzing their ions in the gas phase which lies beyond the scope of traditional analytical methods.
\end{abstract}

Cycloparaphenylenes (CPPs) are attractive model compounds as they constitute the smallest elements of carbon nanotubes (CNTs) and "seed particles" for a bottom-up fabrication of CNTs. ${ }^{[1]}$ Furthermore, their electronic and optoelectronic properties qualify CPPs as active components of molecular electronics and photovoltaics. ${ }^{[2]}$ CPPs with sizes from 5 to 16 and 18 phenylene rings have been synthesized. ${ }^{[3]}$ With increasing number of phenylenes, different CPPs are hardly separable via conventional methods and have escaped further analysis.

Similar to the first pioneering synthesis of [n]CPPs, our synthetic approach (Scheme 1) is based on Suzuki coupling of 1,4-syn-dimethoxy-2,5-cyclohexadiene derivatives $\mathbf{1}$ and $\mathbf{2}$ as "kinked" precursors. ${ }^{[4]}$ The molecules are prone of macrocyclization as shown in previous works. ${ }^{[5]}$ We extended the reaction time to 96 hours to ensure complete conversion and introduced phenyl groups as end-cappers to achieve a better distinction of cyclic and linear products. The resulting mixture 3 was separated from insoluble material which we attribute to the formation of acyclic oligomers. Reductive aromatization of the cyclic products 3 by treatment with sodium naphthalenide afforded a mixture of fully conjugated CPPs. Their insolubility prevents separation by chromatographic methods and characterization by solution NMR or single crystal X-ray diffraction.

[a] Dr. W. Zhang, A. Abdulkarim, Dr. F. E. Golling ${ }^{\dagger}$, Dr. H. J. Räder, Prof. Dr. K. Müllen

Max Planck Institute for Polymer Research

Ackermannweg 10, 55128, Mainz (Germany)

E-mail: raeder@mpip-mainz.mpg.de muellen@mpip-mainz.mpg.de.

${ }^{\dagger}$ Present address: Covestro AG, 51365, Leverkusen, Germany

Supporting information for this article is given via a link at the end of the document.
Herein, we elucidate the complex mixture using matrixassisted laser desorption ionization (MALDI) together with ion mobility mass spectrometry (IMMS). These methods combine three features: i) solvent-free sample preparation of insoluble analytes, ii) molecular size dependent separation by IMS, and iii) tandem mass spectrometry. The analysis points toward homoand heterocatenanes where only "macro-mono-cycles" would have been expected.

High resolution MALDI MS using our solvent-free sample preparation $^{[6]}$ (Figure 1) discloses signals up to $6390 \mathrm{Da}$. The mass difference between neighboring monoisotopic peaks is $456.19 \mathrm{Da}$, corresponding to the molecular weight of six paraphenylene groups. The detected absolute masses correspond exactly to multiples of $456.19 \mathrm{Da}$, indicating the absence of end groups. Thus, the oligomer peaks can be assigned exclusively to CPPs consisting of equal numbers of the initial A-A and B-B type building blocks with ring sizes from $12,18,24 \ldots$ up to 84 phenylene rings. A CPP with 6 phenylene units is not detected which can be rationalized by its high ring strain. The absence of odd numbered repeat units, such as oligomers with 3,9 , 15, etc. phenylene rings, further supports the assignment to cyclic structures, because those are not possible from the underlying chemistry. The homologous series of molecules is denoted as $P_{n}$ where " $n$ " represents the number of phenylene rings. High resolution and exact mass measurements enable the assignment of elemental compositions for each oligomer. As shown for the signal at 2737.1323 Da (insert in Figure 1), the chemical formula is $\mathrm{C}_{216} \mathrm{H}_{144}$ and the experimental isotopic distribution fits well to theory, thus matching a CPP consisting of 36 phenylene units, here denoted as $P_{36}$.

IMS can separate gas phase ions of equal molecular weight due to their molecular shape differences, represented by their collision cross-section (CCS) values. Even isomeric species can be distinguished, thereby overcoming the intrinsic limitation of mass spectrometry to separate ions exclusively due to their

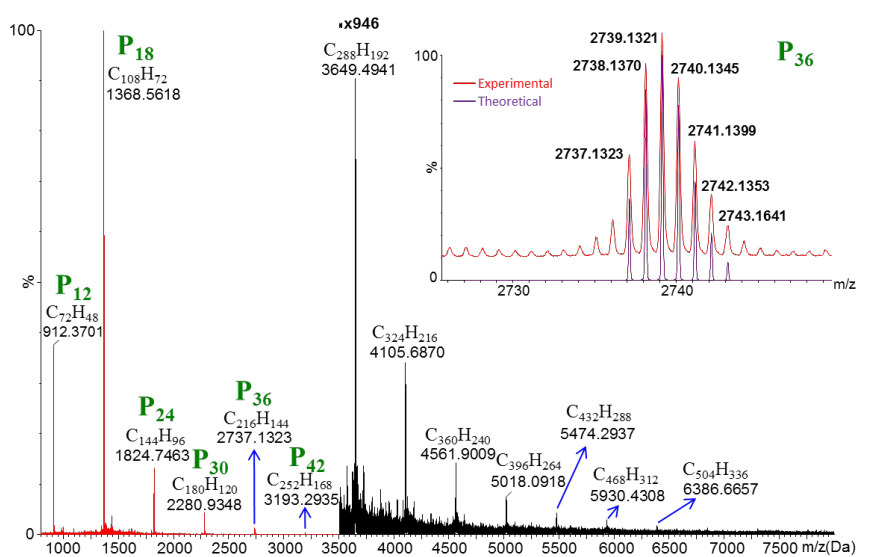

Figure 1. MALDI mass spectrum of the insoluble CPP product mixture. 


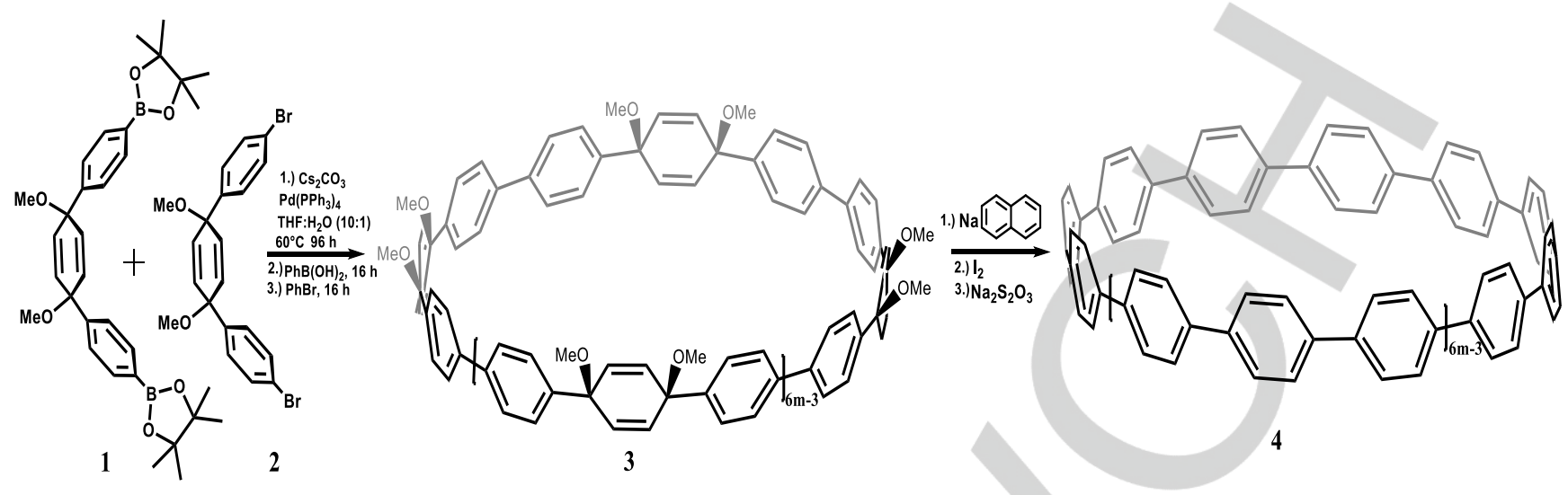

mass-to-charge $(\mathrm{m} / \mathrm{z})$ ratio. $^{[7]}$ So far, IMS has been used to reveal the molecular architectures of a series of mechanically interlocked polyrotaxanes ${ }^{[8]}$, catenanes $^{[9]}$ and polymers ${ }^{[10]}$.

Figure 2 shows the ion mobility spectra of oligomers up to $P_{36}$. The measurements are carried out by using $10 \mathrm{~V}$ collision energy prior to ion mobility separation in order to dissociate noncovalently bonded aggregates of small CPPs which also contribute to a small extend to the signals $P_{24}, P_{30}$ and $P_{36}$ (Figure S1). Therefore, the ion mobility traces in Figure 2 are exclusively due to covalently and mechanically bonded isomers. The ion mobility spectra of the small oligomers $\mathrm{P}_{12}, \mathrm{P}_{18}, \mathrm{P}_{24}$ and $P_{30}$ show only one peak in each case, suggesting that there is only one structural isomer present. They can be assigned to increasing ring sizes, i.e. [12]-, [18]-, [24]- and [30]CPPs. The IMS trace of $\mathrm{P}_{36}$, however, displays four clearly visible signals with significant drift time (DT) differences pointing towards, at least, four different isomeric structures. From the continuous increase in drift time with increasing CPP size of the homologous series in Figure 2, we assign the signal with the highest drift time to the macrocycle with 36 phenylene rings. The peaks at lower drift times, however, must be due to molecules with considerably more compact molecular shapes, i.e. smaller CCS values.

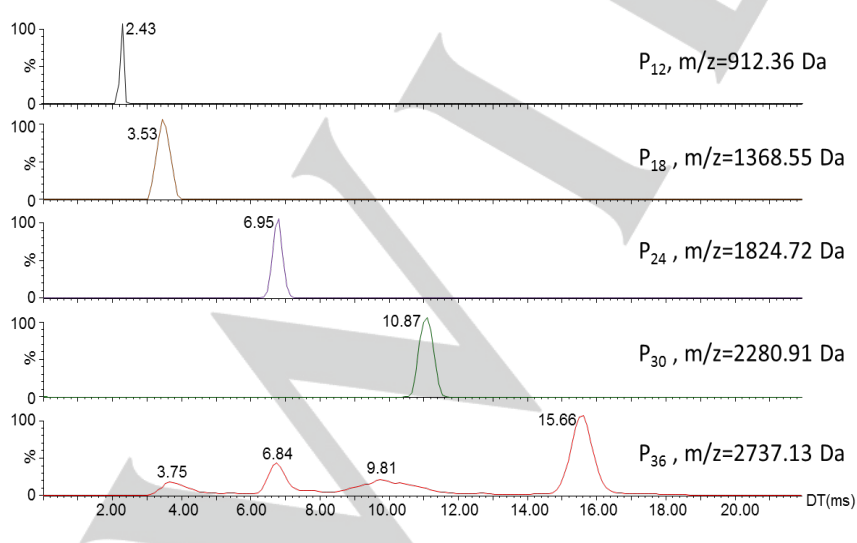

Figure 2. Ion mobility spectra of different CPP oligomers obtained at very low collision energy of $10 \mathrm{~V}$ to remove non-covalently bonded aggregates (see $\mathrm{SI}$ ).
Application of CID to the isomers of $\mathrm{P}_{36}$ after ion mobility separation provides insight into the individual fragmentation behavior (Figure S2). In Figure 3, the intact isomeric structures of $\mathrm{P}_{36}$ are denoted from M1 to M4 according to their drift time differences. The dissociation products own the same drift time as the precursor structures inside the green frames.

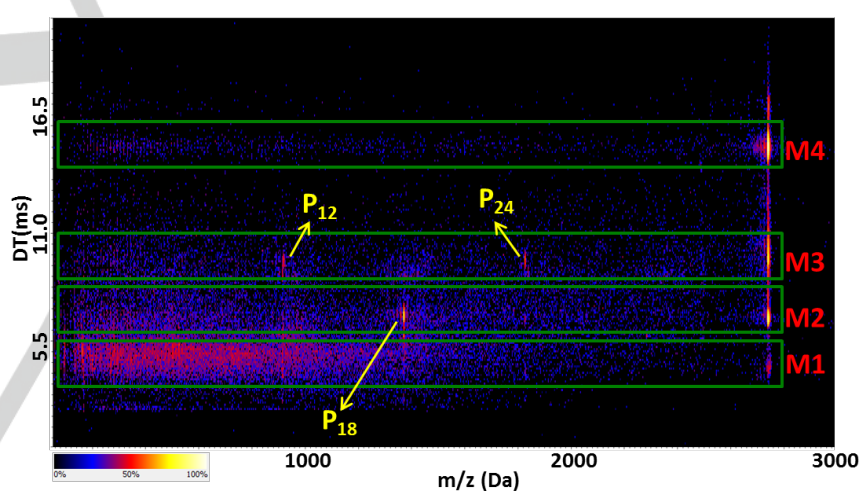

Figure 3. IMMS image of $P_{36}$ obtained with CID at $200 \mathrm{~V}$ after ion mobility separation. The $x$ - axis corresponds to the $m / z$ value and the $y$ - axis represents the drift time. The brightness of the color indicates the peak intensity (yellow implies high intensity).

In case of $\mathrm{M} 3$, two dissociation products appear at $912 \mathrm{Da}$ (annotated as $\mathrm{P}_{12}$ ) and $1814 \mathrm{Da}$ (annotated as $\mathrm{P}_{24}$ ). These $\mathrm{m} / \mathrm{z}$ values correspond exactly to [12]CPP and [24]CPP, therefore suggesting that M3 consists of two smaller macrocycles with different sizes. Analogously, there is only one intense peak at $1312 \mathrm{Da}\left(\mathrm{P}_{18}\right)$ with the same drift time as its parent ion M2 This indicates that M2 consists of two [18]CPPs. We thus safely conclude that the isomeric structures originate from catenanes with mechanically interlocked CPPs, where at least one covalent bond has to be broken to cause a dissociation into cyclic and linear fragment ions, both having exactly the same $\mathrm{m} / \mathrm{z}$ values. M4 does not produce any fragments at $200 \mathrm{~V}$ which is in accordance with the assignment to the regular macrocycle [36]CPP. In case of M1, the smallest component of the $P_{36}$ 
isomer mixture, no dissociation into defined masses of smaller ring sizes can be observed. The absence of defined fragments and the compact structure (smaller than $2 \times[18] \mathrm{CPP}$ ) suggest the presence of a molecular knot $^{[9]}$ of the [36] CPP. Furthermore, it can be speculated that the high signal width in case of M1 and M3 arises from conformational flexibility of certain isomers as well as from contributions of more complicated interlocked structures of the catenanes.

In summary, the results from fragment ion analysis of $P_{36}$ provide clear evidence for the existence of two different catenanes, exclusively composed of either one [12] CPP and one [24] CPP or two [18] CPPs as presented in Figure 4. Estimated from the peak intensities of $\mathrm{P}_{36}$ isomers, the catenanes occur in significant amounts as compared to the regular [36]CPP.

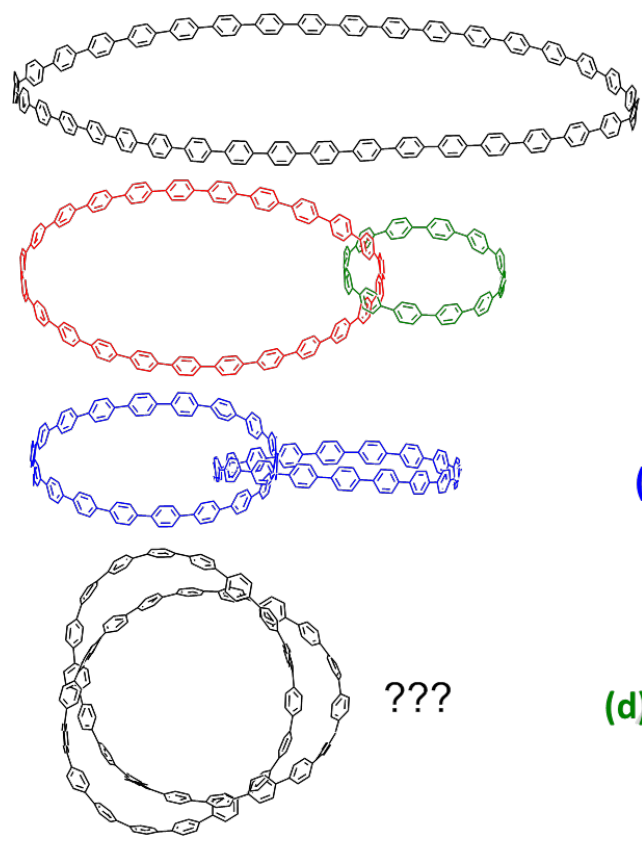

(a) [36]CPP

(b) [12]CPP $+[24]$ CPP (c) $2 \times[18] \mathrm{CPP}$

Figure 4. Isomeric structures of macrocycle, catenanes and trefoil knot hidden in $\mathrm{P}_{36}\left(\mathrm{C}_{216} \mathrm{H}_{144}\right.$, exact mass $\left.=2737.1323 \mathrm{Da}\right)$.

The assignment to catenanes with different compositions can be rationalized by molecular modeling. Therefore, the threedimensional (3D) structures of the four $\mathrm{P}_{36}$ isomers with different topologies are energy optimized by molecular dynamics calculations $^{[11]}$ and submitted to CCS value calculations (Experimental part in SI). The derived CCS values provide a relative size determination which can be qualitatively compared to the measured drift times in IMS. Ions with larger CCS values at the same molecular weight move slower in ion mobility separation, hence having higher drift times. The calculated CCS values are summarized in Table 1.

The size decrease, represented by the decrease of CCS values in the order of [36]CPP $\left(904 \AA^{2}\right)$, [2]catenane ([12]CPP+[24]CPP) $\left(878 \AA^{2}\right)$, [2]catenane $(2 \times[18] \mathrm{CPP})\left(841 \AA^{2}\right)$ and the trefoil knot of [36]CPP $\left(655.01 \AA^{2}\right)$, is in line with the compositions and the decreasing drift times detected from M4 to M1.

Table 1. Calculated CCS values for the (single chain) macrocycle and potential catenane as well as trefoil structures of $P_{36}$.

\begin{tabular}{lllll}
\hline & {$[36] \mathrm{CPP}$} & $\begin{array}{l}\text { [2]catenane } \\
([12] \mathrm{CPP}+[24] \mathrm{CPP})\end{array}$ & $\begin{array}{l}\text { [2]catenane } \\
(2 \times[18] \mathrm{CPP})\end{array}$ & Trefoil knot \\
\hline $\begin{array}{lllll}\text { CCS } \\
\text { value } \\
\left(\AA^{2}\right)\end{array}$ & $\begin{array}{l}\text { (Figure } \\
\text { 4a) }\end{array}$ & $\begin{array}{l}878 \\
\text { (Figure 4b) }\end{array}$ & $\begin{array}{l}841 \\
\text { (Figure 4c) }\end{array}$ & $\begin{array}{l}655.01 \\
\text { (Figure 4d) }\end{array}$ \\
\hline
\end{tabular}

Thus, ion mobility tandem mass spectrometry of an insoluble oligomeric mixture of CPPs reveals an unexpected, but exciting structural complexity. Whereas the results from traditional mass spectrometry would have been erroneously assigned to pure macrocycles up to very large ring sizes with 84 phenylene units, our findings demonstrate the co-existence of macrocycles and catenanes. Even though this has been proven for isomeric structures at a mass of $2737 \mathrm{Da}$, it can be expected that the larger macrocycles are in reality even more complex mixtures of catenanes and knots rather than ordinary monocycles.

From a synthetic viewpoint, the formation of significant amounts of CPP catenanes without targeted approaches of supramolecular preorganization ${ }^{[12]}$ of the phenylene building blocks is certainly surprising and deserves further attention.

\section{Acknowledgements}

This work was supported by the European Research Council grant on NANOGRAPH, Graphene Flagship (No. CNECT-ICT604391), European Union Projects UPGRADE and MoQuas (contract N.610449).

Keywords: Cycloparaphenylene $\cdot$ Ion mobility mass spectrometry $\cdot$ Catenane $\cdot$ Molecular Knot $\bullet$ Macrocycle

[1] aR. Jasti, C. R. Bertozzi, Chemical physics letters 2010, 494, 1-7; bH. Omachi, T. Nakayama, E. Takahashi, Y. Segawa, K. Itami, Nature chemistry 2013, 5; cH. Omachi, Y. Segawa, K. Itami, Accounts of chemical research 2012, 45, 13781389.

[2] aX. Guo, M. Baumgarten, K. Müllen, Progress in Polymer Science 2013, 38, 1832-1908; bC. Li, M. Liu, N. G. Pschirer, M. Baumgarten, K. Müllen, Chemical reviews 2010, 110, 6817-6855; cM. R. Talipov, A. Boddeda, Q. K. Timerghazin, R. Rathore, The journal of physical chemistry. $C$, Nanomaterials and interfaces 2014, 118, 2140021408 . 
[3] E. R. Darzi, R. Jasti, Chemical Society Reviews 2015, 44, 6401-6410.

[4] R. Jasti, J. Bhattacharjee, J. B. Neaton, C. R. Bertozzi, Journal of the American Chemical Society 2008, 130, 17646-17647.

[5] aE. R. Darzi, T. J. Sisto, R. Jasti, The Journal of Organic Chemistry 2012, 77, 66246628; bF. E. Golling, M. Quernheim, M. Wagner, T. Nishiuchi, K. Müllen, Angewandte Chemie International Edition 2014, 53, 1525-1528; cM. Quernheim, F. E. Golling, W. Zhang, M. Wagner, H.-J. Räder, T. Nishiuchi, K. Müllen, Angewandte Chemie International Edition 2015, 54, 10341-10346; dF. E. Golling, S. Osella, M. Quernheim, M. Wagner, D. Beljonne, K. Müllen, Chemical Science 2015, 6, 7072-7078.

[6] aL. Przybilla, J.-D. Brand, K. Yoshimura, H. J. Räder, K. Müllen, Analytical chemistry 2000, 72 , 4591-4597; bK. Yoshimura, L. Przybilla, S. Ito, J. D. Brand, M. Wehmeir, H. J. Räder, K. Müllen, Macromolecular Chemistry and Physics 2001, 202, 215-222; cS. Trimpin, S. Keune, H. J. Räder, K. Müllen, Journal of the American Society for Mass Spectrometry 2006, 17, 661-671.

[7] aA. B. Kanu, P. Dwivedi, M. Tam, L. Matz, H. H. Hill Jr, Journal of Mass Spectrometry 2008, 43, 122; bR. Mukhopadhyay, Analytical chemistry 2008 , 80 .

[8] aG. De Bo, J. De Winter, P. Gerbaux, C.-A. Fustin, Angewandte Chemie International Edition 2011, 50, 9093-9096; bC. a. Scarff, J. R. Snelling, M. M.

Knust, C. L. Wilkins, J. H. Scrivens, C. A. Scar, Journal of the American Chemical Society 2012, 134, 9193-9198.

[9] C. A. Schalley, J. Hoernschemeyer, X. Li, G. Silva, P. Weis, International Journal of Mass Spectrometry 2003, 228, 373-388.

[10] aA. Yol, C. Wesdemiotis, Reactive and Functional Polymers 2014, 80; bK. Guo, Z. Guo, J. M. Ludlow, T. Xie, S. Liao, G. R. Newkome, C. Wesdemiotis, Macromolecular rapid communications 2015, 36, 1539-1552; cW. Zhang, M. Quernheim, H. J. Räder, K. Müllen, Analytical Chemistry 2016, 88, 952-959.

[11] M. D. Hanwell, D. E. Curtis, D. C. Lonie, T. Vandermeersch, E. Zurek, G. R. Hutchison, Journal of cheminformatics 2012, 4, 17-17.

[12] aM. Weck, B. Mohr, J.-P. Sauvage, R. H. Grubbs, The Journal of Organic Chemistry 1999, 64, 54635471; bG. Gil-Ramírez, D. a. Leigh, A. J. Stephens, Angewandte Chemie International Edition 2015, 54, 6110-6150. 


\section{COMMUNICATION}

Solvent-free MALDI ion mobility mass spectrometry is used to analyze an insoluble product mixture of cycloparaphenylenes. For the first time, monocycles and isomeric catenanes can be detected in a simple reaction mixture, where only monocycles could be expected.

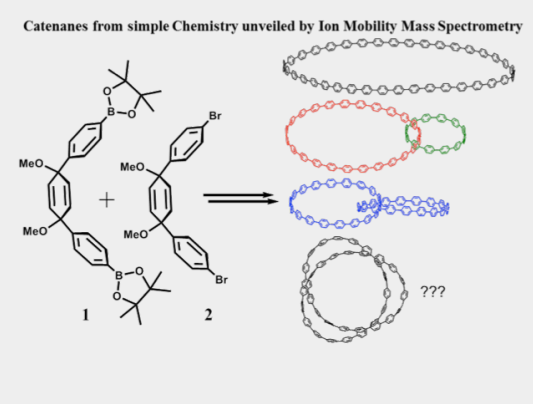

Wen Zhang, Ali Abdulkarim, Florian E. Golling, Hans Joachim Räder*, and Klaus Müllen*

\section{Page No. 1- Page No.4}

Cycloparaphenylenes and their Catenanes - Complex Macrocycles Unveiled by Ion Mobility Mass Spectrometry 